\title{
El debate sobre la importancia del LDL colesterol y uso de estatinas
}

\author{
Alexis Lama Toro \\ Cardiólogo
}

El tratamiento con estatinas para disminuir el LDL colesterol sanguíneo ha sido el pilar de la cardiología preventiva en los últimos años, llevando a algunos investigadores a proponer esta terapia a toda persona $>50$ años, e incluso a los niños de $\geq 8$ años con LDL colesterol aumentado. Las recientes guías americanas para el manejo del colesterol sanguíneo recomiendan en pacientes $\leq 75$ años con riesgo no muy alto de enfermedad ateroesclerótica, como en aquellos con alto riesgo, un tratamiento de alta intensidad con estatinas, lo que quiere decir, dosis máximas, 40- $80 \mathrm{mg}$ de atorvastatina o 20 - $40 \mathrm{mg}$ de rosuvastatina, con la meta de reducir el colesterol $\mathrm{LDL} \geq 50 \%$, y personas con LDL- colesterol $\geq 190 \mathrm{mg} / \mathrm{dl}$, independiente del riesgo cardiovascular $(\mathrm{CV})$ deberían recibir estatinas, también en dosis de alta intensidad.

Las guías taiwanesas, por su parte, recomiendan para pacientes con síndrome coronario agudo (SCA) y enfermedad coronaria, estatinas o estatinas más ezetimibe en todos los pacientes con SCA, teniendo como meta bajar el LDL colesterol a $<70 \mathrm{mg} / \mathrm{dl}$ y a $<55 \mathrm{mg} / \mathrm{dl}$ si, además, tienen diabetes.

El reciente Panel de Consenso de la Sociedad Europea de Ateroesclerosis también aboga por la necesidad de re- ducir intensamente el LDL colesterol, con el objeto de lograr una disminución de la enfermedad cardiovascular ateroesclerótica (ECVAE), ya que, concluyen, que el LDL colesterol es causa (no una simple asociación) de la ECVAE1.

Todas estas recomendaciones, y también las de otras numerosas guías, se fundamentan obviamente en una copiosa e importante bibliografía, la que de acuerdo a sus autores, constituye evidencia científica innegable y absolutamente irrefutable, la que por supuesto es seguida - a veces con matices dogmáticos - por un gran número de médicos en el mundo entero y en nuestro país.

Sin embargo, hay que reconocer que desde hace muchos años existe una creciente controversia en relación con el verdadero rol que le corresponde al LDL- colesterol en la ECVAE y, actualmente, me parece que ésta se ha magnificado. El número de investigadores de diferentes partes del mundo, sin conflictos de intereses con la Industria farmacéutica, que desafían y cuestionan, con sus correspondientes argumentos, la importancia atribuida al LDL colesterol en la ECVAE y el uso intensivo de estatinas para disminuir el riesgo de ella, está claramente en alza, con lo que el debate al respecto parece francamente estar 
al rojo, y se manifiesta con ácidas acusaciones cruzadas, reciprocas y, también, en numerosas y algunas recientes publicaciones, que critican el fundamento de las recomendaciones expresadas anteriormente, $\mathrm{e}$ intentan invalidarlas por falta de solidez científica ${ }^{2,3,4}$.

Se esgrime, por ejemplo, entre los muchos argumentos, que si el LDL colesterol fuese realmente la causa de ECVAE, necesaria y lógicamente, tendría que siempre estar elevado en pacientes con ECVAE, no tratados. Pero un estudio realizado en 136.905 pacientes con infarto agudo de miocardio encontró que, al momento de la admisión, éstos tenían LDL colesterol sanguíneo considerados bajos (casi la mitad con valores $<100 \mathrm{mg} / \mathrm{dl})^{2}$.

Por otra parte, si el colesterol LDL fuese en verdad aterogénico, como se cree, lógicamente personas con elevado nivel de LDL colesterol tendrían que exhibir más ateroesclerosis que aquellas con baja concentración de LDL colesterol. Y aunque hay un estudio que sí encontró asociación entre un nivel elevado de LDL colesterol y ateroesclerosis subclínica, hay varios otros que no encontraron tal asociación ${ }^{2}$. Por otro lado, si el LDL colesterol es causa de ECVAE y de mayor mortalidad, parecería lógico que esto se reflejase en todos los grupos poblaciones, sin embargo, no es así. El estudio epidemiológico más grande realizado en Japón reveló que todas las causas de mortalidad fueron inversamente correlacionadas con los niveles de LDL colesterol, tanto en hombres como en mujeres 5 .

Otro de los puntos controvertidos, es el efecto de las estatinas sobre la mortalidad. De hecho, existen 44 estudios clínicos controlados al azar, poco difundidos, que no mostraron beneficio en disminuir mortalidad, y varios de ellos tampoco mostraron reducción de eventos cardiovasculares ${ }^{4}$. Otros autores han reportado que las estatinas podrían, a lo más, sólo posponer la muerte en una media de 3.1 días y 4.2 días, en prevención primaria y secundaria, respectivamente. El reciente estudio ACCELERATE, controlado, al azar, tampoco demostró una reducción importante en eventos CV o mortalidad, a pesar de un aumento de $130 \%$ en HDL colesterol y una disminución de 37\% en LDL colesterol. También se cuestionan los potenciales beneficios del uso de estatinas en reducir la mortalidad por enfermedad coronaria, considerados como exagerados, ya que se ha recurrido a mostrar los resultados en función de riesgos relativos y no absolutos, aparte de omitir en los estudios presentados, varios estudios clínicos, haciendo ver, además, que en los estudios realizados después del año 2005, en que las autoridades europeas y americanas introdujeran nuevas regulaciones para los estudios clínicos, especificando que los datos de las investigaciones deban ser públicos, los beneficios de las estatinas han, virtualmente, desaparecido. Se critica además, la afirmación de que "la mayor evidencia para causalidad está dada por más de 30 estudios clínicos controlados al azar que, consistentemente, han demostrado que reducir el LDL colesterol reduce el riesgo de eventos $\mathrm{CV}$ de una manera proporcional a la reducción absoluta en el LDL colesterol", ya que la figura que respalda esta conclusión incluye datos sólo de 12 de los 33 estudios citados y que al reconstruir una nueva figura, incluyendo los 21 estudios omitidos, se observa que no hay asociación entre la disminución del LDL colesterol y el número de eventos coronarios $^{2,3}$.

Finalmente, es interesante conocer en relación con la discontinuación de la terapia con estatinas, que, aunque hay estudios que muestran que puede provocar un aumento del riesgo de cardiopatía isquémica fatal, hay otros autores que niegan esta posibilidad, afirmando que la suspensión de ellas no ha conllevado un aumento en la mortalidad $\mathrm{CV}^{6}$.

En síntesis, creo que no se puede seguir haciendo caso omiso de los cuestionamientos que se hacen en la actualidad a la importancia del LDL colesterol y al uso de estatinas en ECVAE, de los cuales sólo he mostrado algunos y de manera sucinta. Creo que es importante abrirse al debate, confrontar y analizar los distintos puntos de vista, con el único objetivo de acercarnos a la verdad y, con ello, ofrecer la mejor evidencia científica a nuestros pacientes. 


\section{Referencias}

1. FERENCE B, GINSBERG H, GRAHAM I, RAY K, PACKARD C, BRUCKERT E, et al. Low-density lipoproteins cause atherosclerotic cardiovascular disease. 1. Evidence from genetic, epidemiologic, and clinical studies. A consensus statement from the European Atherosclerosis Society Consensus Panel. Eur Heart J. 2017 Aug 21; 38:2459-2472.

2. RAVNSKOV U, DE LORGERIL M, DIAMOND D, HAMA R, HAMAZAKI T, HAMMARSKJÖLD B, et al. LDL-C does not cause cardiovascular disease: a comprehensive review of the current literature. Expert Rev Clin Pharmacol. 2018;11:959-970.

3. OKUYAMA H, HAMAZAKI T, HAMA R, OGUSHI Y, KOBAYASHI T, OHARA N, et al. A Critical Review of the Consensus
Statement from the European Atherosclerosis Society Consensus Panel 2017. Pharmacology. 2018; 101:184-218.

4. DUBROFF R. Cholesterol paradox: a correlate does not a surrogate make. Evid Based Med. 2017; 22:15-19.

5. HAMAZAKI T, OKUYAMA H, OGUSHI Y, HAMAR. Towards a Paradigm Shift in Cholesterol Treatment. A Re-examination of the Cholesterol Issue in Japan. Ann Nutr Metab. 2015; 66:1-116.

6. RABAEUS M, NGUYEN P, DE LORGERIL M. Recent Flaws in Evidence-Based Medicine: Statin Effects in Primary Prevention and Consequences of Suspending the Treatment. J Controversies Biomed Res. 2017; 3: 1-10. 